\title{
Band Structure of the Growth Rate of the Two-Stream Instability of an Electron Beam Propagating in a Bounded Plasma
}

\author{
I. D. Kaganovich ${ }^{a 1}$ and D. Sydorenko ${ }^{b 1}$ \\ ${ }^{a}$ Princeton Plasma Physics Laboratory, Princeton University, Princeton, New Jersey 08543, \\ USA \\ ${ }^{b}$ University of Alberta, Edmonton, Alberta T6G 2E1, Canada
}

\begin{abstract}
This paper presents a study of the two-stream instability of an electron beam propagating in a finite-size plasma placed between two electrodes. It is shown that the growth rate in such a system is much smaller than that of an infinite plasma or a finite size plasma with periodic boundary conditions. Even if the width of the plasma matches the resonance condition for a standing wave, a spatially growing wave is excited instead with the growth rate small compared to that of the standing wave in a periodic system. The approximate expression for this growth rate is $\gamma \approx(1 / 13) \omega_{p e}\left(n_{b} / n_{p}\right)\left(L \omega_{p e} / v_{b}\right) \ln \left(L \omega_{p e} / v_{b}\right)\left[1-0.18 \cos \left(L \omega_{p e} / v_{b}+\pi / 2\right)\right]$, where $\omega_{p e}$ is the electron plasma frequency, $n_{b}$ and $n_{p}$ are the beam and the plasma densities, respectively, $v_{b}$ is the beam velocity, and $L$ is the plasma width. The frequency, wave number and the spatial and temporal growth rates as functions of the plasma size exhibit band structure. The amplitude of saturation of the instability depends on the system length, not on the beam current. For short systems, the amplitude may exceed values predicted for infinite plasmas by more than an order of magnitude.
\end{abstract}

PACS numbers: 52.35.Qz, 52.40.Mj, 52.65.-y, 52.77.-j

\section{INTRODUCTION}

Interaction of electron beams with plasmas is of considerable importance for many plasma applications where electron emission occurs from surfaces. The electrons extracted from the surface and accelerated in the sheath form a beam of electrons; the beam propagating in the plasma excites electron plasma waves through the twostream instability $\stackrel{1}{\underline{1}}$ Laboratory plasmas and plasmas in industrial applications are usually bounded by electrodes. We show that electrodes greatly affect the growth of the two-stream instability. Though beam-plasma systems have been studied extensively in the past using kinetic simulations, $\underset{-2}{-5}$ the presence of electrically connected boundaries changes the character of the twostream instability from convective to absolute, similar to the instability of a Pierce diode ${ }^{6}$ In the Pierce diode, the instability was studied extensively taking only beam electrons and neutralizing ions into account as relevant to vacuum diodes, see e.g. Ref. 7 and the references within. Here, we consider the two-stream instability between a low density electron beam and high density plasma electrons as relevant to discharges. In this Letter, we have performed an analytical study and fluid and particle-incell simulations in order to obtain the growth rate of the two-stream instability in a finite plasma bounded by electrically connected electrodes. To the best of our knowledge and to some extent to our surprise the solution to this problem was not reported before.

The linear stage of the instability can be described making use of fluid formalism which includes the continuity equations

$$
\frac{\partial n_{e, b}}{\partial t}+\frac{\partial v_{e, b} n_{e, b}}{\partial x}=0
$$

the momentum equations

$$
\frac{\partial v_{e, b}}{\partial t}+v_{e, b} \frac{\partial v_{e, b}}{\partial x}=-\frac{e}{m} E
$$

and the Poisson equation

$$
\frac{\partial^{2} \phi}{\partial x^{2}}=4 \pi e\left(n_{e}+n_{b}-n_{i}\right),
$$

where $n_{e, b}$ and $v_{e, b}$ are the densities and the velocities of the plasma and beam electrons, $-e$ and $m$ are the electron charge and mass, $E=-\partial \phi / \partial x$ is the electric field, $\phi$ is the electric potential, and $n_{i}$ is the ion density. The initial plasma state is neutral: $n_{e, 0}+n_{b, 0}=n_{i, 0}$, where $n_{e, 0}$ and $n_{b, 0}$ are the initial densities of the bulk and the beam electrons, and $n_{i, 0}$ is the initial density of ions, respectively. The ion density is uniform and constant, $n_{i}=n_{i, 0}=$ const. Initially, the bulk and the beam electron densities and the beam flow velocity are uniform everywhere. Note that everywhere in this paper subscripts $e$ and $b$ denote values related to plasma and beam electrons, respectively.

For the studies described in the present paper, the boundary conditions are non-periodic and describe a plasma produced in a discharge between two electrodes. At the ends of the system $x=0$ and $x=L$, the potential perturbations are set to zero, $\phi(0)=\phi(L)=0$. The beam is injected at the boundary $x=0$. The boundary conditions for the beam electrons are $n_{b}(0)=n_{b, 0}$ and $v_{b}(0)=v_{b, 0}$, where $v_{b, 0}$ is the injection velocity of the beam. Note that in fluid simulations, a small sheath forms near the electrodes and more accurate boundary conditions are required to account for the sheath effect. 8

The paper is organized as follows. In Section III, a dispersion relation for the finite-length beam-plasma system is derived. In Section III complex frequencies and wavenumbers obtained by direct solution of the dispersion equation are compared with the fluid simulation 
and approximate analytical formulas for the frequency, wavenumber, and temporal and spatial growth rates are given. Section IV compares growth rates in kinetic simulations with the predictions of the fluid theory. Section $\mathrm{V}$ discusses the amplitude of saturation of the instability and provides analytical formulas for the estimate of the saturation electric field amplitude. The results are summarized in Section VI.

\section{ANALYTICAL SOLUTION}

The dispersion equation is obtained by solving linearized Eqs. (1] 3) for perturbations of plasma and beam electron densities and velocities. The perturbations are defined as

$$
\begin{aligned}
\delta n_{e} & =n_{e}-n_{e, 0}, \delta n_{b}=n_{b}-n_{b, 0} \\
\delta v_{b} & =v_{b}-v_{b, 0}, \delta v_{e}=v_{e}
\end{aligned}
$$

Linearized equations can be readily solved using Laplace's method. 9 However, we are only looking for an asymptotic solution which the system approaches on longer times. Following the Pierce method, $\underline{\underline{6}}$ the asymptotic solution for the potential has the following form:

$$
\delta \phi(t, x)=\left(A x+B e^{i k_{+} x}+C e^{i k_{-} x}+D\right) e^{-i \omega t},
$$

where $\omega$ is the frequency of the wave, $k_{ \pm}$are the wave vectors of the two waves propagating in the system, and coefficients $A, B, C, D$ are complex constants. The density and the velocity perturbations are

$$
\begin{aligned}
\delta n_{e, b}(t, x) & =\left(\delta n_{e, b}^{\prime}+\delta n_{e, b}^{+} e^{i k_{+} x}+\delta n_{e, b}^{-} e^{i k_{-} x}\right) e^{-i \omega t} \\
\delta v_{e, b}(t, x) & =\left(\delta v_{e, b}^{\prime}+\delta v_{e, b}^{+} e^{i k_{+} x}+\delta v_{e, b}^{-} e^{i k_{-} x}\right) e^{-i \omega t}
\end{aligned}
$$

The linearized equations for the parts of the perturbations proportional to $\exp \left(-i \omega t+i k_{ \pm} x\right)$ are

$$
\begin{aligned}
-i \omega \delta n_{e}^{ \pm}+i k_{ \pm} \delta v_{e}^{ \pm} n_{e, 0} & =0 \\
-i \omega \delta v_{e}^{ \pm} & =\frac{e}{m} i k_{ \pm} \delta \phi^{ \pm}, \\
-i \omega \delta n_{b}^{ \pm}+i k_{ \pm}\left(\delta v_{b}^{ \pm} n_{b, 0}+v_{b, 0} \delta n_{b}^{ \pm}\right) & =0 \\
\left(-i \omega+i k_{ \pm} v_{b, 0}\right) \delta v_{b}^{ \pm} & =\frac{e}{m} i k_{ \pm} \delta \phi^{ \pm}, \\
-k_{ \pm}^{2} \delta \phi^{ \pm} & =4 \pi e\left(\delta n_{e}^{ \pm}+\delta n_{b}^{ \pm}\right),
\end{aligned}
$$

where $\delta \phi^{+}=B$ and $\delta \phi^{-}=C$. These equations yield:

$$
\begin{aligned}
& \delta v_{e}^{ \pm}=\frac{\omega}{k} \frac{\delta n_{e}^{ \pm}}{n_{e, 0}}, \frac{\delta n_{e}^{ \pm}}{n_{e, 0}}=-\frac{e}{m} \frac{k_{ \pm}^{2}}{\omega^{2}} \delta \phi^{ \pm} \\
& \delta v_{b}^{ \pm}=\frac{\omega-v_{b, 0} k_{ \pm}}{k_{ \pm}} \frac{\delta n_{b}^{ \pm}}{n_{b, 0}}, \frac{\delta n_{b}^{ \pm}}{n_{b, 0}}=-\frac{e}{m} \frac{k_{ \pm}^{2}}{\left(\omega-k v_{b}^{ \pm}\right)^{2}} \delta \phi^{ \pm}
\end{aligned}
$$

Substitution of relations (6) into the Poisson equation gives usual dispersion relation for waves

$$
1=\frac{\omega_{e, 0}^{2}}{\omega^{2}}+\frac{\omega_{b, 0}^{2}}{\left(\omega-v_{b, 0} k_{ \pm}\right)^{2}} .
$$

Here $\omega_{e, 0}^{2} \equiv 4 \pi e^{2} n_{e, 0} / m$ and $\omega_{b, 0}^{2} \equiv 4 \pi e^{2} n_{b, 0} / m$ are the electron plasma frequencies corresponding to the plasma and beam densities.

The uniform parts of the density and velocity perturbations (5), which are proportional to $\exp (-i \omega t)$ and correspond to high-frequency uniform electric field given by the first term in Eq. (4), are obtained in a similar way:

$$
\delta v_{e}^{\prime}=\delta v_{b}^{\prime}=\frac{i e}{\omega m} A, \delta n_{e}^{\prime}=\delta n_{b}^{\prime}=0 .
$$

These perturbations correspond to high-frequency current flowing through the plasma and allow for $\delta v_{e} \neq 0$ at the systems ends; $\delta v_{b}(0)=0$ because beam is injected with a given velocity but $\delta v_{b}(L) \neq 0$.

Applying four boundary conditions $\delta n_{b}(0)=\delta v_{b}(0)=$ $\delta \phi(0)=\delta \phi(L)=0$ to perturbations (4) and (5) and taking into account (6) and (7) in the form

$$
\omega-k_{ \pm} v_{b, 0}= \pm \frac{\omega_{b, 0}}{\sqrt{1-\frac{\omega_{e, 0}^{2}}{\omega^{2}}}}
$$

gives the following additional relation between $\omega$ and $k$ :

$$
\begin{gathered}
k_{-}^{2}\left(e^{i k_{+} L}-1\right)-\frac{i k_{-}^{2} k_{+} \omega L}{\omega-k_{+} v_{b, 0}}= \\
k_{+}^{2}\left(e^{i k_{-} L}-1\right)-\frac{i k_{+}^{2} k_{-} \omega L}{\omega-k_{-} v_{b, 0}} .
\end{gathered}
$$

Eqs. (9) and (10) determine the temporal $[\operatorname{Im}(\omega)]$ and the spatial $[\operatorname{Im}(k)]$ growth rates of the instability as well as the frequency $[\operatorname{Re}(\omega)]$ and the wavenumber $[\operatorname{Re}(k)]$. If plasma electrons are absent and only beam electrons are taken into account $\left(n_{e, 0}=0\right)$, Eq. (10) reduces to the Pierce's dispersion relation for vacuum diode.

In order to solve the dispersion relation (10), we introduce a new dimensionless variable

$$
\chi=\frac{\omega_{b, 0} / \omega_{e, 0}}{\sqrt{1-\frac{\omega_{e, 0}^{2}}{\omega^{2}}}} .
$$

Substituting (11) into (9) and assuming that $\omega=\omega_{e, 0}$ in the left-hand side of (9) gives

$$
k_{ \pm}=(1 \mp \chi) \frac{\omega_{e, 0}}{v_{b, 0}} .
$$

Substitution (12) into (10) yields equation for $\chi$

$$
\begin{gathered}
-i \frac{2(1-\chi)}{(1+\chi) \chi} L_{n}+e^{i(1-\chi) L_{n}}-1- \\
\frac{(1-\chi)^{2}}{(1+\chi)^{2}}\left[e^{i(1+\chi) L_{n}}-1\right]=0
\end{gathered}
$$


where $L_{n} \equiv L \omega_{e, 0} / v_{b, 0}$ is the normalized gap width.

Equation (13) gives $\chi$ as a function of $L_{n}$. The frequency is calculated from (11) and for a low-density beam with $n_{b, 0} \ll n_{e, 0}$ it is

$$
\omega=\frac{\omega_{e, 0}}{\sqrt{1-\frac{\omega_{b, 0}^{2}}{\omega_{e, 0}^{2} \chi^{2}}}} \approx \omega_{e, 0}\left(1+\frac{n_{b, 0}}{2 n_{e, 0} \chi^{2}}\right) .
$$

The wavenumbers $k_{ \pm}$can be obtained from (12).

Function $\chi\left(L_{n}\right)$ is complex with band structure, i.e. it changes abruptly at certain $L_{n}=c+2 \pi l$, where $c$ is a constant and $l$ is an integer. Indeed, in the limit of $L_{n} \gg 1$, the first two terms in (13) are dominant which gives the following approximate expression:

$$
-i \chi L_{n} e^{-i \chi L_{n}}=2 L_{n}^{2} e^{-i L_{n}} .
$$

Here, we used the fact that $|\chi| \ll 1$ and $\operatorname{Im}(\chi)>0$. The solution of this equation is the Lambert or productlog function: ${ }^{10}$

$$
-i \chi L_{n}=W\left(2 L_{n}^{2} e^{-i L_{n}}\right)
$$

This function has many branches, the branch selected must ensure the maximal growth rate. When parameters of the plasma, e.g. the discharge gap, change, a transition from one branch to another may occur and the instability growth rate will change abruptly.

Since $\chi$ is complex and independent on $n_{b, 0}$, it follows from (14) that the temporal growth rate of the instability is proportional to $\omega_{e, 0}\left(n_{b, 0} / n_{e, 0}\right)$ unlike the growth rate of the resonant perturbation $k \approx \omega_{e, 0} / v_{b, 0}$ in a periodic system proportional to $\omega_{e, 0}\left(n_{b, 0} / n_{e, 0}\right)^{1 / 3_{1}}$.

The analytical solution is verified by fluid and particlein-cell (PIC) simulations described below.

\section{FLUID SIMULATIONS}

The fluid numerical model solves Eqs. (1)-(3). The densities in (11) are advanced using the SHASTA method $\stackrel{11}{=}$ The velocities in (2) are advanced using an upwind scheme $\frac{12}{n}$ The model demonstrates excellent agreement with the theory $\underline{\underline{1}}$ in simulations of the instability of a cold beam in a cold plasma with periodic boundary conditions.

The fluid simulations are carried out with the following common parameters: $n_{e, 0}=2 \times 10^{17} \mathrm{~m}^{-3}, \omega_{e, 0}=$ $2.52 \times 10^{11} \mathrm{~s}^{-1}$, beam energy $50 \mathrm{eV}$ and beam velocity $v_{b, 0}=4.2 \times 10^{6} \mathrm{~m} / \mathrm{s}$, the numerical grid cell size is $1.3 \mu \mathrm{m}$, the time step is $0.9 \mathrm{fs}$. The selected values of spatial and temporal steps ensure stability of the SHASTA algorithm. The resonant beam wavelength $\lambda_{b} \equiv 2 \pi v_{b, 0} / \omega_{e, 0}$ is $1.044 \mathrm{~mm}$ for these plasma parameters. Initially, the bulk electron flow velocity is given a harmonic perturbation $\delta v_{e}=\delta v_{e, 0} \sin \left(x \omega_{e, 0} / v_{b, 0}\right)$ with

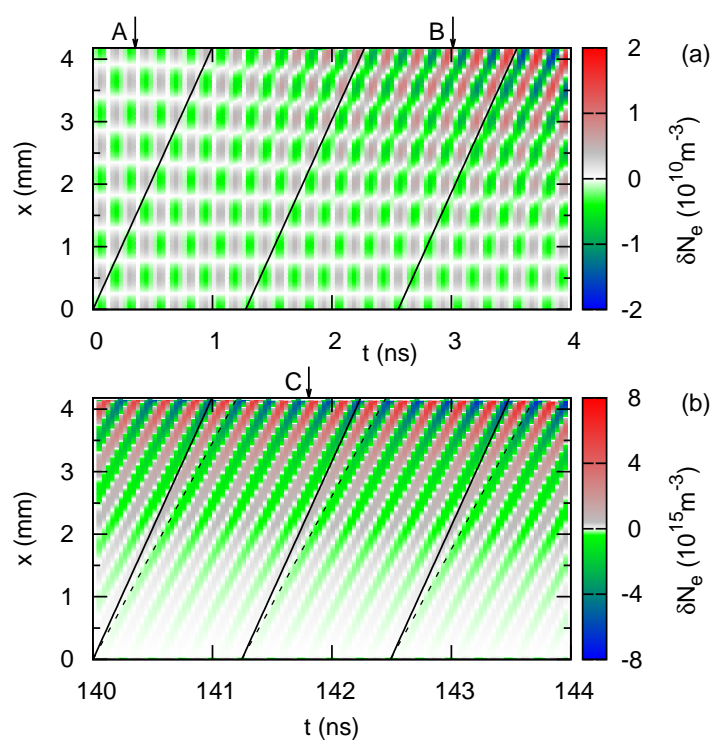

FIG. 1. Evolution of the bulk electron density perturbation in time and space in fluid simulation with $L=4 \lambda_{b}$ and $\alpha=0.0006$. Panels (a) and (b) correspond to the very beginning of the fluid simulation (a) and to the asymptotic constant growth stage (b); the corresponding temporal growth of the electric field amplitude is shown by the red curve in Fig. 3 . Solid black lines in (a) and (b) represent propagation with the unperturbed beam velocity. Dashed black lines in (b) represent phase velocity of the wave calculated as $\operatorname{Re}(\omega) / \operatorname{Re}(k)$, where $\operatorname{Re}(\omega)=2.522 \times 10^{10} \mathrm{~s}^{-1}$ and $\operatorname{Re}(k)=7.288 \mathrm{~mm}^{-1}$. Arrows A, B, and C mark times $t_{A}=0.35 \mathrm{~ns}, t_{B}=3.01 \mathrm{ns,}$ and $t_{C}=141.8 \mathrm{~ns}$ when profiles shown in Figs. 2a, 20 $\mathrm{b}$, and 2r. are obtained.

the wavelength corresponding to the resonance in a periodic or an infinite plasma, the amplitude of the perturbation is very small, $\delta v_{e, 0}=0.1 \mathrm{~m} / \mathrm{s}$.

The oscillations have the wavelength of the initial perturbation during only the first few periods. The initial oscillation pattern corresponds to a standing wave. As the instability develops, the standing wave transforms to a propagating wave, see Fig. 17. This process is accompanied by the shrinking of the wavelength, compare the density perturbation profiles at three consecutive times in Fig. 2. At the initial phase of the instability, the perturbations propagate with the original beam velocity, see Fig. 113. At the asymptotic stage given by Eq.(4) with the spatial growth rate along the beam propagation, the wave phase velocity is noticeably lower than the velocity of beam propagation, compare the slope of the black dashed line with that of the black solid lines in Fig. 1b.

Simulation reveals that before the asymptotic state establishes, the temporal growth rate changes with time, see Fig. 3. Initially, the growth rate is large compared to the analytical value defined by Eqs. (14) and (13). Then it gradually decreases towards the asymptotic value predicted by the theory and it stays approximately constant for tens and even hundreds of plasma periods until the 

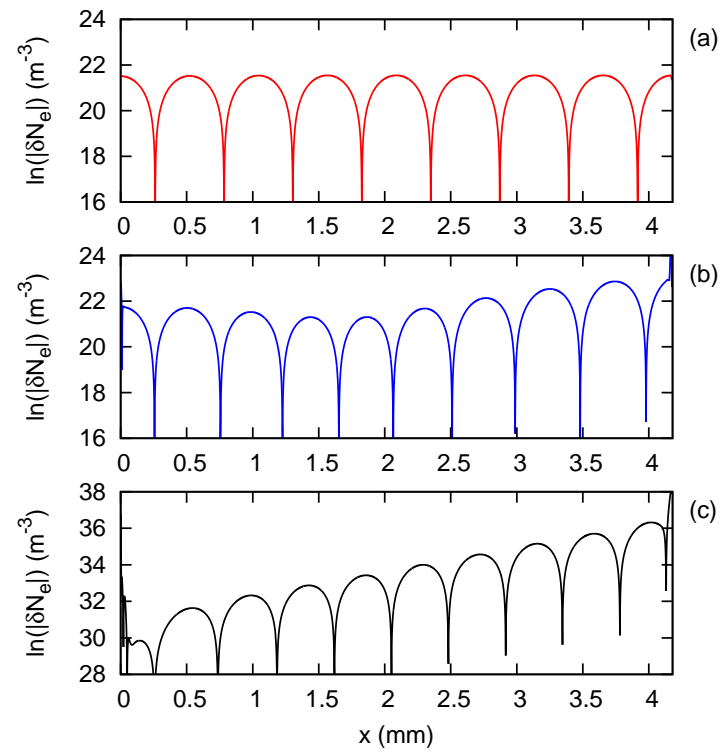

FIG. 2. Spatial profiles of bulk electron density perturbation obtained at $t_{A}=0.35 \mathrm{~ns}(\mathrm{a}), t_{B}=3.01 \mathrm{~ns}(\mathrm{~b})$, and $t_{C}=$ $141.8 \mathrm{~ns}(\mathrm{c})$. Times $t_{A, B, C}$ are shown by arrows A, B, and C in Fig. 1.

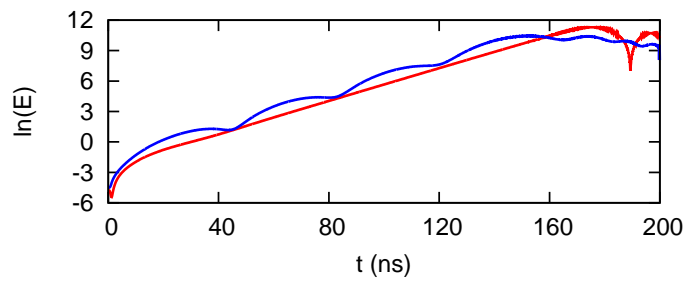

FIG. 3. Amplitude of electric field oscillations versus time in fluid simulations with $\alpha=0.0006$ and $L=4 \lambda_{b}$ (red curve), $L=4.7 \lambda_{b}$ (blue curve). The curves are obtained in the point with coordinate $x=3.55 \mathrm{~mm}$ (red) and $x=4.34 \mathrm{~mm}$ (blue).

nonlinear stage of instability and its saturation occurs, see the red curve in Fig. 3. Note that the modification of the wavelength mentioned above stops when the instability reaches the asymptotic stage, which for the red curve in Fig. 3 occurs near 20 ns.

In order to investigate the dependence of the growth rate on plasma parameters, four simulation sets are discussed below. In set one, the ratio of the beam to plasma density is $\alpha \equiv n_{b, 0} / n_{p, 0}=0.00015$, the size of the system $L$ increases from $\lambda_{b}$ to $8.5 \lambda_{b}$. Set two is similar to set one but the beam density is higher, $\alpha=0.0006$. In set three, $L=3.4 \lambda_{b}$ is constant while $\alpha$ changes from 0.0001 to 0.0006 . The fourth set is similar to set three but $L=8.3 \lambda_{b}$.

In all simulations, the growth rates, the frequencies, and the wavenumbers are calculated during the asymptotic stage when the temporal growth rate is constant for a prolonged period of time, see the red curve in Fig. 3 for $20 \mathrm{~s}<t<160 \mathrm{~s}$. In simulation sets one and two, for some values of $L$ such a stage never appears, see the blue curve in Fig. 3. These values of $L$ correspond to the gaps in the simulation data seen in Fig. 4 .

Overall, there is an excellent agreement between the simulations and the theory. The dimensionless values of $\left[\operatorname{Re}(\omega)-\omega_{e, 0}\right] /\left(\omega_{e, 0} \alpha\right), \operatorname{Im}(\omega) /\left(\omega_{e, 0} \alpha\right), \operatorname{Re}\left(k \lambda_{b}\right)$, and $\operatorname{Im}\left(k \lambda_{b}\right)$ obtained in simulation sets one and two (red and black curves in Fig. 4) and by analytical solution of the theoretical dispersion relation (blue crosses in Fig. 4) are very close to each other and appear to be functions of the dimensionless system length only, as predicted by the analytical solution given by Eqs. (12) and (14). These functions for $\operatorname{Re}(\omega), \operatorname{Im}(\omega)$, and $\operatorname{Re}(k)$ have band structure. Mathematically, it is the consequence of the presence of many branches in the Lambert function. The instability growth is given by the maximum growth rate value that changes from branch to branch when the gap size crosses some critical value, typically when $L / \lambda_{b}$ approaches an integer, see Fig. 4. Similar band structure was also observed for the Pierce diode $\stackrel{7,13}{ }$ Figure 41 shows the number of wave periods in the gap as a function of the gap length. In all cases, it is very close to an integer number, although not exactly:

$$
\operatorname{Re}(k) L /(2 \pi) \simeq\left\lceil L / \lambda_{b}\right\rceil,
$$

where $\lceil x\rceil \equiv \operatorname{ceiling}(x)$ is the smallest integer not less than $x$.

Since the shape of the functions is universal for various beam densities, it is reasonable to introduce approximate formulas which fit the numerical solution as follows:

$$
\begin{gathered}
\operatorname{Re}(\omega) \approx \frac{\omega_{e, 0} \alpha}{18} L_{n} \ln \left(L_{n}\right)\left[1-0.9 \cos \left(L_{n}+0.4\right)\right] \\
\operatorname{Im}(\omega) \approx \frac{\omega_{e, 0} \alpha}{13} L_{n} \ln \left(L_{n}\right)\left[1-0.18 \cos \left(L_{n}+\frac{\pi}{2}\right)\right] \\
\operatorname{Re}(k) \approx \frac{\omega_{e, 0}}{v_{b, 0}}\left[1.1+\frac{1+2.5 \cos \left(L_{n}\right)}{1.1 L_{n}}\right] \\
\operatorname{Im}(k) \approx \frac{\omega_{e, 0}}{v_{b, 0}} \frac{2 \ln \left(L_{n}\right)-0.5}{L_{n}}
\end{gathered}
$$

The wavenumber and the spatial growth rate depend on the system length but are virtually insensitive to the beam density, see Fig. 55 and Fig. 5d. The temporal growth rate is approximately linearly proportional to the relative beam density $\alpha$. The linear law holds especially well for short systems, see the red curve in Fig. [5] and compare red and black curves for $L / \lambda_{b}<6$ in Fig. 4 b. For longer systems, however, deviation from the linear law becomes noticeable.

\section{TEMPORAL GROWTH RATE IN KINETIC SIMULATION}

Kinetic simulations are carried out with the EDIPIC 1D3V particle-in-cell (PIC) code ${ }^{14}$ The code is modified 

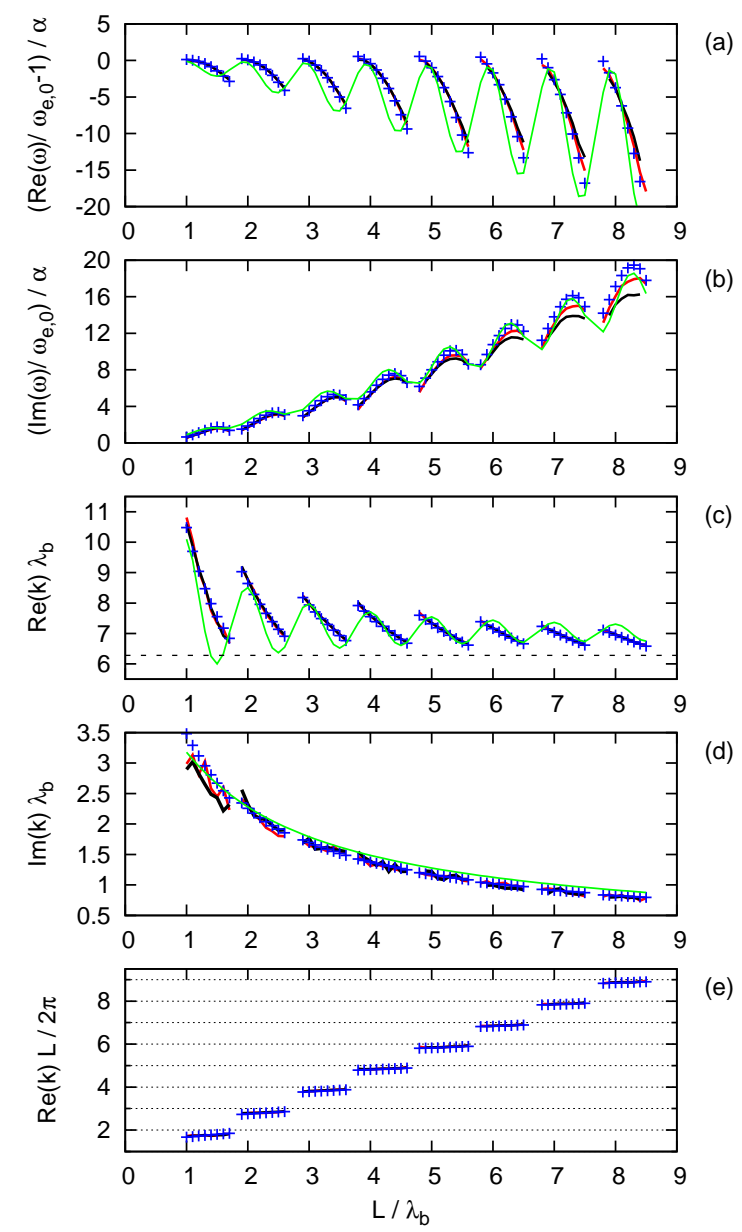

FIG. 4. Frequency (a), temporal growth rate (b), wavenumber (c), spatial growth rate (d), and the number of wave periods per system length (e) versus the length of the system. The blue crosses mark values obtained by analytical solution given by equations (13), (14), and (12). Solid red and black curves represent values obtained in fluid simulations with $\alpha=0.00015$ (red) and $\alpha=0.0006$ (black). Solid green curves are values provided by fitting formulas (17), (18), (19), and (20). In (c), the black dashed line marks the resonant wavenumber.

to reproduce conditions of the fluid simulations. The ions form an immobile background, the boundaries have zero potential. The bulk electrons are reflected specularly from the boundaries. The beam electrons penetrate through the boundaries freely. The initial plasma density and the beam energy are the same as in the fluid simulations. Collisions are omitted. Two simulations are carried out with $L=8.3 \lambda_{b}, \alpha=0.0006$ but different number of particles per cell. One simulation has 10000 particles per cell. The other simulation has 2000 particles per cell. Below these simulations are referred to as $10 \mathrm{k}$ and $2 \mathrm{k}$ simulations, respectively.

PIC simulations start with a significant level of statistical noise which is few orders of magnitude higher than the initial perturbation induced in the fluid simulations
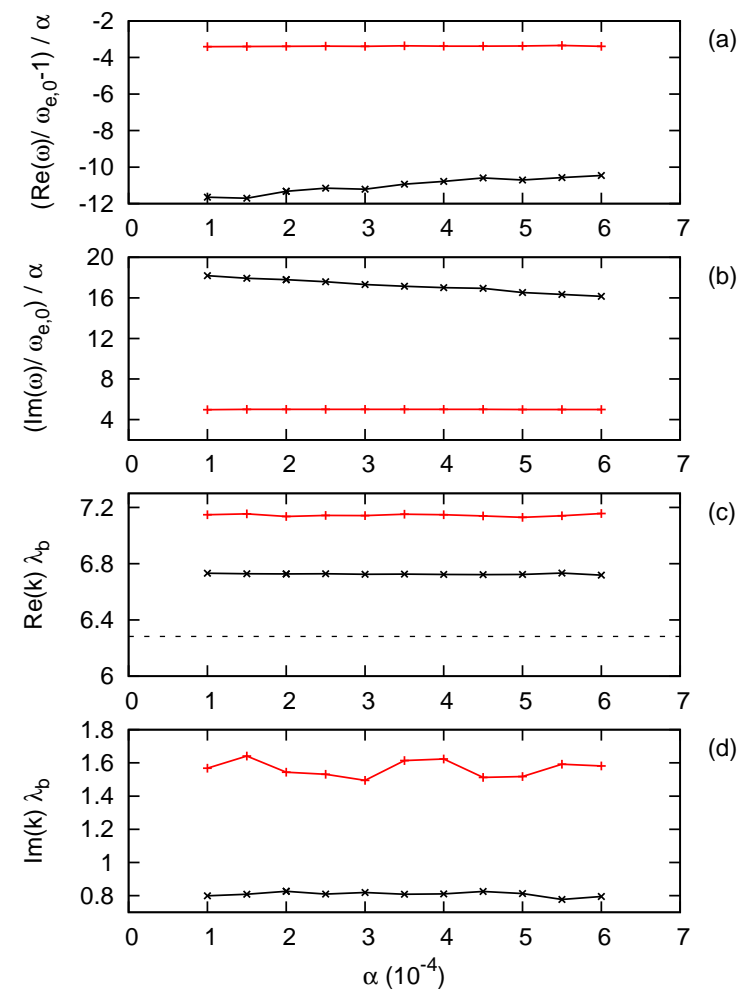

FIG. 5. Frequency (a), temporal growth rate (b), wavenumber (c), and spatial growth rate (d) versus the ratio of the beam and bulk electron densities in fluid simulations with $L=3.4 \lambda_{b}$ (red) and $L=8.3 \lambda_{b}$ (black). In (c), the black dashed line marks the resonant wavenumber.

above. At the same time, the amplitudes of nonlinear saturation of the instability in PIC and fluid simulations are close to each other. As result, the time when the oscillations grow from the initial noise level to the saturation in PIC simulation is much shorter than that in a fluid simulation. Moreover, at the initial stage the growth rate gradually decreases which furthermore limits the duration of the asymptotic stage described by analytic solution. For example, in the $10 \mathrm{k}$ simulation, the asymptotic stage lasts from $10 \mathrm{~ns}$ to $20 \mathrm{~ns}$ while in the fluid simulation that stage occurs between $10 \mathrm{~ns}$ and 45 ns, compare the green and the red curves in Fig. 6. The short asymptotic stage in the 10k simulation still allows to calculate the temporal growth rate which appeared to be very close to the value obtained in fluid simulations. In the $2 \mathrm{k}$ simulation, however, the noise level is higher and the asymptotic stage is very short and barely detectable, see the blue curve in Fig. [6]

\section{SATURATION AMPLITUDE IN KINETIC SIMULATION}

PIC simulations described below are carried out with the following common parameters. The initial uniform 


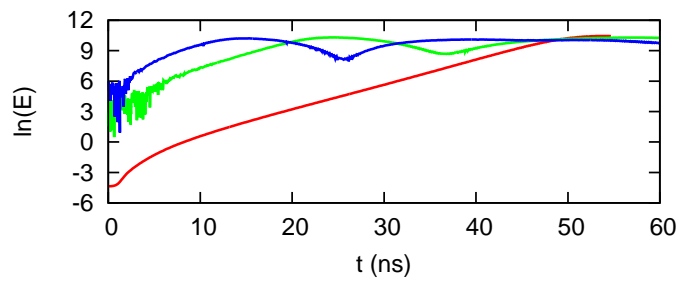

FIG. 6. Amplitude of electric field oscillations vs time in simulations with $L=8.3 \lambda_{b}$ and $\alpha=0.0006$. The curves represent fluid simulation (red), PIC simulation with 10000 particles per cell (green), and PIC simulation with 2000 particles per cell (blue).

plasma electron density is $n_{e, 0}=2 \times 10^{17} \mathrm{~m}^{-3}$, the initial electron beam energy or the energy of injection is $W_{b}=50 \mathrm{eV}$, the beam-to-plasma density ratio is $\alpha=1.5 \times 10^{-4}$, the initial plasma electron temperature $T_{e, 0}=0.5 \mathrm{eV}$, the size of a cell of the computational grid is $\Delta x=2.078 \times 10^{-6} \mathrm{~m}$ corresponding to $\lambda_{D, e} / 8$ where $\lambda_{D, e}$ is the electron Debye length of the ambient plasma, both the plasma and the beam initially are represented by 2500 macroparticles per each cell of the grid. The ions are represented by an immobile uniform background with density which ensures that the plasma-beam system is initially neutral. The electron beam propagates in the positive $x$-direction.

The following three PIC simulations are carried out. First simulation has periodic boundary conditions and the system length of $L=2 \lambda_{b}$ where $\lambda_{b}=2 \pi V_{b} / \omega_{e, 0}$ is the wavelength of the plasma wave resonant with the beam in an infinite plasma, $V_{b}$ is the beam velocity. For the selected parameters, $\lambda_{b}=1.044 \mathrm{~mm}$. Second simulation has non-periodic boundary conditions similar to the ones used in the fluid model. The boundaries are grounded, the plasma electrons are reflected from the boundaries while the beam electrons penetrate through them freely. System length in the second simulation is $L=8.3 \lambda_{b}$ which corresponds to the maximum of the temporal growth rate in the dispersion band with $L / \lambda_{b} \approx 8$ in the fluid simulation. Third simulation also has the nonperiodic similar to the second simulation, but the system length is much shorter, only $L=1.5 \lambda_{b}$. In the second and the third simulations, the beam injection occurs at the boundary $x=0$.

The theory of interaction of a cold beam with a cold plasma predicts that the exponential growth of the amplitude of plasma oscillations is followed by saturation and subsequent amplitude oscillations $\frac{15}{\underline{5}}$ Such a picture is reproduced in the first simulation, see Figs. 7(a) and (c). The plasma wave propagates in the direction of beam propagation and has constant amplitude along the system, see Fig. Z(b) and Fig. 8(a). The theoretical growth rate is

$$
\operatorname{Im}(\omega)=0.7 \alpha^{1 / 3} \omega_{e, 0}
$$

and the electric field amplitude in the first maximum is

$$
E_{1, \max }=3 k W_{b} \alpha^{2 / 3}
$$

where $\omega_{e, 0}$ is the Langmuir frequency of plasma electrons and $k=\omega_{e, 0} / V_{b}$ is the resonance wavenumber. For the selected beam and plasma parameters, $\omega_{e, 0}=2.52 \times$ $10^{10} \mathrm{~s}^{-1}, V_{b}=4.19 \times 10^{6} \mathrm{~m} / \mathrm{s}$, and $k=6015.9 \mathrm{~m}^{-1}$. Therefore, the theoretical growth rate (21) is

$$
\operatorname{Im}(\omega)=0.938 \times 10^{9} \mathrm{~s}^{-1}
$$

and the electric field amplitude maximum (22) is

$$
E_{1, \max }=2549 \mathrm{~V} / \mathrm{m} \text {. }
$$

Both values are very close to the simulation results, see the red curve in Fig. 7(c) and compare it with the dashed straight line which corresponds to the theoretical growth rate (21). It is necessary to mention here that during the first $4 \mathrm{~ns}$, the growing oscillations are obscured by the noise present in the system due to the finite number of particles in simulation. The saturation begins at $t=6$ ns when the beam particles start passing each other, see the phase plane in Fig. 8 (b). Note that by this time the beam electrons in the laboratory frame travel about 25 $\mathrm{mm}$ which is several times more than the length of the system in the second simulation.

In the second simulation, the boundary conditions are non-periodic and the linear stage of the instability follows the fluid theory for the finite-length systems developed above - the wave amplitude grows both along the system and in time, the temporal growth rate is close to the fluid value, compare the red curve with the straight black dashed line in Fig. $7(\mathrm{~g})$. The saturation of the amplitude occurs around $70 \mathrm{~ns}$ and here the amplitude is maximal near the exit end of the system, see Fig. 7(d) and (f) near arrow 1 and the electric field profile in Fig. 8(c). The maximum wave amplitude is an order of magnitude higher than that in the periodic simulation, and it causes much stronger velocity perturbations of the beam particles. Due to both limited distance of interaction between the beam and the wave and the nonuniform wave amplitude, the beam electrons start passing each other only near the exit end, see Fig. 8 (d).

An interesting process occurs after the first saturation. Position of the maximum amplitude gradually moves towards the injection boundary until it reaches the distance of about $5.5 \mathrm{~mm}$, and the value of the maximum almost doubles, see Fig. 7(d) and Fig. 8(e). After $150 \mathrm{~ns}$, it is this new maximum where the passing of beam electrons is achieved, not the exit end of the system, see Fig. 8(f). Downstream of this maximum $(x>6 \mathrm{~mm})$ the beam electrons are completely mixed in the phase plane. As a result of this change in the beam structure, while upstream of the maximum $(x<5 \mathrm{~mm})$ the wave propagates along the beam direction with spatially growing amplitude, downstream of the maximum the wave pattern is closer to that of a standing wave, compare Figs. $7(\mathrm{e})$ and (f). 

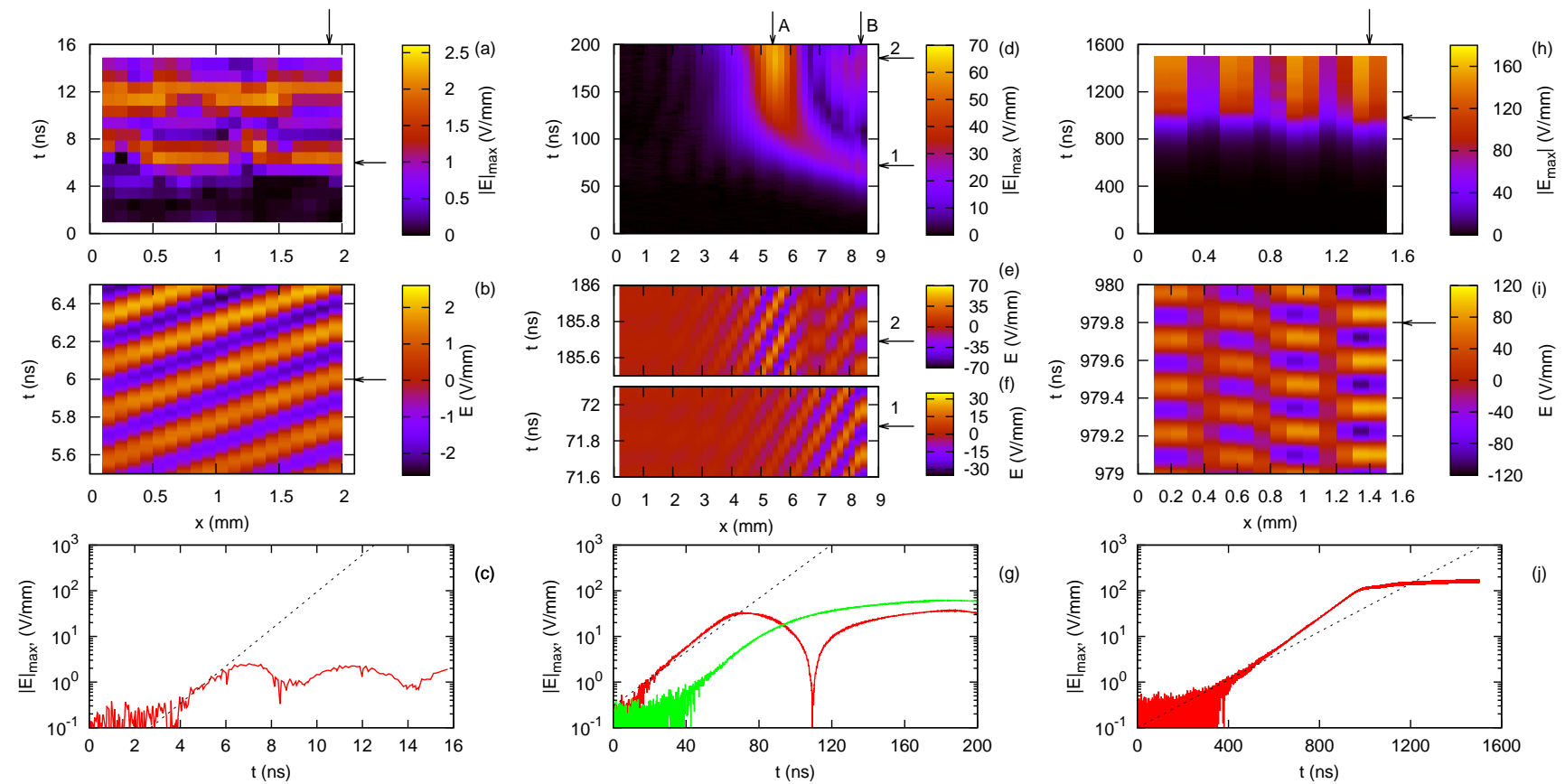

FIG. 7. Results of PIC simulation with periodic boundaries (a,b,c), non-periodic boundaries and $L=8.3 \lambda_{b}$ (d,e,f,g), and non-periodic boundaries and $L=1.5 \lambda_{b}(\mathrm{~h}, \mathrm{i}, \mathrm{j})$. The top row (a,d,h) shows amplitude of oscillations versus coordinate and time. The middle row (b,e,f,i) shows electric field versus coordinate and time. The bottom row (c,g,j) shows the amplitude of oscillations versus time at certain locations marked by vertical arrows in (a), (d), and (h), respectively. The green and the red curves in (g) correspond to locations marked by vertical arrows A and B in (d), respectively. The horizontal arrows in (a,b) mark time of the snapshot shown in Figs. 8(a,b). The horizontal arrows in (d,e,f) mark time of snapshots shown in Figs. 8 $(c, d)$ (arrow 1) and in Figs. 8(e,f) (arrow 2). The horizontal arrows in (h,i) mark time of the snapshot shown in Figs. 8(g,h). The dashed black straight line in (c) shows the exponential growth with the theoretical growth rate in an infinite plasma. The dashed black straight lines in $(\mathrm{g})$ and $(\mathrm{j})$ corresponds to the growth rates obtained in fluid simulations with the same system length and beam current.

The second simulation clearly shows that the amplitude of saturation of the two stream instability in finite length plasmas can be significantly higher than that in an infinite plasma. In a bounded system, the length of interaction between a beam electron and the plasma wave cannot exceed the distance between the boundaries. An infinite plasma has no such a limit. Here a wave of modest intensity can interact with beam electrons over longer distances before the mixing of the beam electrons in the phase plane occurs. In order to achieve such a mixing over much shorter distances, which is the case in bounded systems, the wave field must be much stronger.

In order to find how strong this effect can be, the third simulation with the non-periodic boundary conditions is performed with a very short system length $L=1.5 \lambda_{b}$. The wave pattern corresponds to a standing wave with 5 nodes and 4 antinodes. The antinodes and the 3 middle nodes are clearly visible in Figs. 7(h) and (i). There are two nodes with approximately zero electric field at the ends of the system. These two nodes were not resolved by the code diagnostics used to produce Figs. $7(\mathrm{~h})$ and (i), but they are visible in the electric field profile in Fig. $8(\mathrm{~g})$. The temporal growth rate in this simulation is about $34 \%$ higher than the growth rate in the fluid simu- lation with the same parameters, compare the red curve with the black straight dashed line in Fig. 7(j(j). The maximal wave amplitude reaches $170 \mathrm{~V} / \mathrm{mm}$ which is more than 60 times stronger than the field in the periodic system, compare Figs. Z7(j) and (c). Such a strong electric field produces mixing of beam electrons on a very short distance of $1 \mathrm{~mm}$ which is about one resonance wavelength, see Fig. 8(h).

The phase plots shown in Fig. 8 prove that in the finite length system the saturation of the instability occurs when the beam particles are overtaking each other. This process depends on the wave amplitude and the system length but should not depend on the beam current. To check this, two additional simulations are carried out with $L=8.3 \lambda_{b}$ and the relative beam density of $\alpha=0.0003$ and 0.0006 . Another two additional simulations with these beam densities are carried out for $L=1.5 \lambda_{b}$. The results of these simulations combined with those obtained above for $\alpha=0.00015$ confirm that the amplitude of the first maximum of saturation of the instability is virtually insensitive to the beam current, see the red and the green curves with markers in Fig. 9(a). The only difference is that for higher current the saturation is achieved faster. Note that in the whole range of 

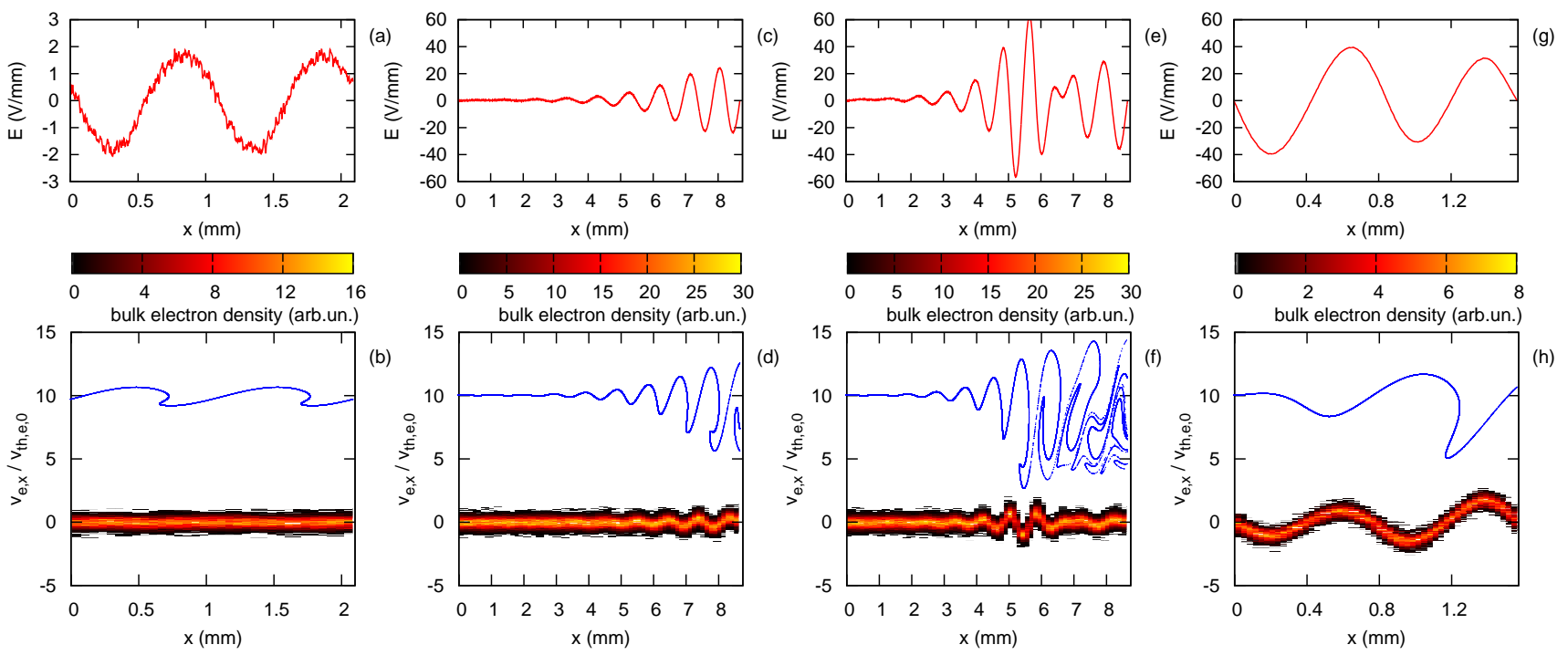

FIG. 8. Electric field profiles (a,c,e,f) and electron "velocity versus coordinate" phase planes (b,d,f,h) in the PIC simulations with periodic boundaries (a,b), non-periodic boundaries and $L=8.3 \lambda_{b}$ (c,d,e,f), and non-periodic boundaries and $L=1.5 \lambda_{b}$ $(\mathrm{g}, \mathrm{h})$. Snapshots $(\mathrm{a}, \mathrm{b})$ are obtained at time $6 \mathrm{~ns}$ shown by the horizontal arrows in Figs. 7(a,b). Snapshots (c,d) are at time $71.88 \mathrm{~ns}$ shown by arrows 1 in Figs. 7(d,f). Snapshots (e,f) are at time 185.69 ns shown by arrows 2 in Figs. 7(d,e). Snapshots $(\mathrm{g}, \mathrm{h})$ are at time $979.8 \mathrm{~ns}$ shown by the horizontal arrows in Figs. 7 (h,i). In the phase planes, the beam electrons are represented by the blue color while the plasma electrons are represented by the color map, the white background correspond to the empty space.

the beam density $\alpha$ considered, the values of the saturation amplitude in the finite system are much higher than the predictions for the infinite system, compare the red and the green curves with markers with the blue curve in Fig. 9(a).

The wave amplitude which causes overtaking of beam particles can be estimated as

$$
\frac{e E}{m_{e}\left(\omega-k v_{b}\right)^{2}} \sim \lambda_{b}
$$

where the left-hand side is the displacement of the particles trapped by the wave in the wave frame. Using equation (12) one can replace $\left(\omega-k v_{b}\right)^{2}$ with $\left(\chi \omega_{e, 0}\right)^{2}$. Then, replacing $\lambda_{b} \omega_{e, 0}$ with $v_{b, 0}$ one can write an expression for the maximal electric field of the wave as

$$
E_{\max }=\frac{m_{e} v_{b, 0} \omega_{e, 0}}{e}\left|\chi^{2}\right| .
$$

For estimates, the value of $\chi^{2}$ is convenient to find as

$$
\chi^{2}=\left\{1-\frac{v_{b, 0}}{\omega_{e, 0}}[\operatorname{Re}(k)+i \operatorname{Im}(k)]\right\}^{2}
$$

with $\operatorname{Re}(k)$ and $\operatorname{Im}(k)$ given by the approximate formulas (19) and (20). Note that expressions (19) and (20) are independent on beam current and are functions of the normalized plasma gap width $L_{n}$ only. Therefore, the maximal field (24) depends on the beam velocity $v_{b}$ and the gap width $L$ but does not depend on the beam current.
A dependence $E_{\max }(L)$ calculated with (25) for the beam parameters used in the simulations above is shown by the black curve in Fig. 9(b). The oscillations in this curve reflect the band structure of the wave number in the finite length system. The saturation values obtained in the PIC simulations are remarkably close to the values given by Eq. (25), compare curves with markers with the horizontal dashed curves of the same color in Fig. 9(a), also compare the markers with the black curve in Fig. 9(b). The value of $E_{\max }$ decreases with $L$ and eventually approaches the saturation values for the infinite system given by Eq. (22), compare the black curve with the horizontal blue lines in Fig. 9(b).

PIC simulations discussed above demonstrate that the growth of the maximal electric field in the two-stream instability in a short system compared to an infinite plasma can be very large. It is necessary to mention, however, that these simulations are carried out with certain simplifications similar to those made in the fluid model. In particular, the sheath is not resolved, the ion background is immobile, and collisions with neutrals are omitted. The realistic sheath will allow some energetic plasma electrons to escape and may affect the structure of the wave interacting with the beam. If the ion dynamics is accounted for, the strong plasma oscillations may result in the modulation instability which will create density cavities and affect the wave. Finally, the two stream instability can be suppressed by electron-neutral collisions if the collision frequency is more than two times the collisionless growth rate. In very short systems, the temporal growth 

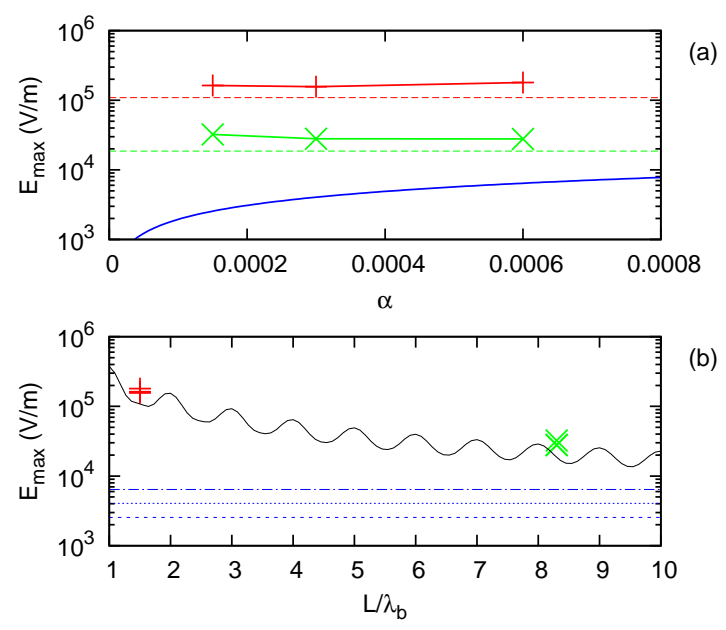

FIG. 9. (a) Amplitude of the first maximum versus the relative beam density in PIC simulations with $L=8.3 \lambda_{b}$ (green) and $L=1.5 \lambda_{b}$ (red). In (a), the solid blue curve is the theoretical prediction (22) for an infinite plasma, the dashed horizontal lines mark theoretical predictions given by Eq. (25) for finite-length systems with $L=8.3 \lambda_{b}$ (green) and $L=1.5 \lambda_{b}$ (red). (b) Saturation amplitude versus the system length. In (b), the black curve is obtained with Eq. (25), the red vertical crosses and the green diagonal crosses mark first amplitude maxima in the PIC simulations with $L=8.3 \lambda_{b}$ (green) and $L=1.5 \lambda_{b}$ (red); the blue horizontal lines mark theoretical values (22) for the infinite plasma with the beam of relative density $\alpha=0.00015$ (dash), 0.0003 (short dash), and 0.0006 (dash-dot). The PIC simulation values for $L=8.3 \lambda_{b}$ and $L=1.5 \lambda_{b}$ are obtained at points marked by vertical arrow B in Fig. 7(d) and by the vertical arrow in Fig. 7(h), respectively.

rate is very small, which means that the neutrals present in a real beam-plasma system may simply prevent the instability from developing.

\section{SUMMARY}

In summary, we have studied the development of the two-stream instability in a finite size plasma bounded by electrodes both analytically and making use of fluid and particle-in-cell simulations. We show that the instability reaches the asymptotic state when the wave structure has the same spatial profile and grows in time with a constant growth rate. The spatial structure of the wave is close to a standing wave but has a spatial growth along the beam propagation. We derived analytic expressions (17,20) for the frequency, wave number and the spatial and temporal growth rates. Obtained analytic solution agrees well with the values given by fluid and particle-in-cell simulations.

The saturation of the instability occurs due to the overtaking of beam particles. Formulas for the estimate of the saturation amplitude (24,25) are derived and are in good agreement with the simulation results. The amplitude of saturation does not depend on the beam current but grows significantly for shorter systems. Compared to the value predicted for an infinite plasma, the saturation amplitude for low-current plasma beam systems of length of a few resonance wavelengths may be higher by more than an order of magnitude.

Acknowledgement: This research was supported in part by U.S. Department of Energy and Air Force Office of Scientific Research. Authors acknowledge valuable discussions with Edward Startsev and Peter Ventzek.

${ }^{1}$ R. Briggs, Electron-Stream Interaction with Plasmas, MIT Press, 1964.

${ }^{2}$ S. Kainer, J. Dawson, R. Shanny, and T. Coffey, Phys. Fluids 15, 493 (1972).

${ }^{3}$ I. J. Morey and R. W. Boswell, Phys. Fluids B 1, 1502 (1989).

${ }^{4}$ H. Gunell, J. P. V. N. Brenning, and S. Torven, Phys. Rev. Lett. 77, 5059 (1996).

${ }^{5}$ P. H. Yoon, T. Rhee, and C.-M. Ryu, Phys. Rev. Lett. 95, 215003 (2005).

${ }^{6}$ J.R. Pierce, J. Appl. Phys. 15, 721 (1944).

${ }^{7}$ A. Piel, "Plasma Physics An introduction to Laboratory, Space and Fusion Plasmas", Springer-Verlag, Berlin 2010.

${ }^{8}$ A. I. Smolyakov, W. Frias, I. D. Kaganovich, and Y. Raitses, Phys. Rev. Lett. 111, 115002 (2013).

${ }^{9}$ M. Rosenbluth, L. Pearlstein and G. Stuart, Phys. Fluids 6, 1289 (1963).

${ }^{10}$ R. M Corless, G. H. Gonnet, D.E.G. Hare, D. J. Jeffrey, D. E. Knuth, Advances in Computational Mathematics 5, 329 (1996).

${ }^{11}$ J. P. Boris, D. L. Book, Journal of Computational Physics 11, 38 (1973).

12" Flux-Corrected Transport: Principles, Algorithms, and Applications", edited by D. Kuzmin, Rainald Löhner, Stefan Turek, Springer, 2005.

${ }^{13}$ J. R. Cary and D. S. Lemons, Journal of Applied Physics 53, 3303 (1982).

${ }^{14}$ D. Sydorenko, Ph.D., University of Saskatchewan (2006).

${ }^{15}$ N. G. Matsiborko, I. N. Onishchenko, and V. D. Shapiro, Plasma Phys. 14, 591 (1972). 